thirteen letters from former pupils who had attended his lectures.

These notebooks implement our knowledge of William Hunter as the foremost teacher of surgical anatomy of his time. His reputation also rests on his immense contribution to midwifery and the assembly of a vast museum of surgical anatomy. This elegantly produced book, with a foreward by Sir Charles Illingworth, would have enhanced his reputation further had it been fully edited, preceded by a biographical introduction and set firmly within the historical context of his times. KenNeth Dewhurst

\section{Particles in the Air}

Assessment of Airborne Particles: Fundamentals, Applications and Implications to Inhalation Toxicity. Edited by Thomas T. Mercer, Paul E. Morrow and Werner Stöber. (A Proceedings Publication of the Third Rochester International Conference on Environmental Toxicity.) Pp. xix +540 . (Charles C. Thomas: Springfield, Illinois, March 1972.) $\$ 23.75$.

THE twenty-seven papers of this volume were presented at a conference held in June 1970 at the Department of Radiation Biology and Biophysics of the School of Medicine and Dentistry, University of Rochester, New York. Five sessions covered aerosol fundamentals, production and measurement of aerosols, size analysis and shape factors, inhaled aerosols and the assessment of hazardous aerosols. There are good author and subject indexes and the book is well produced.

Papers in the first session dealt with the enhancement of rate of coagulation due to polydispersity, the deposition of particles from turbulent flow in a wind tunnel upon artificial grass, impaction of particles upon wires coated with adhesive, the collection efficiency declining as the adhesive became covered with particles, a study of membrane filtration and a survey of existing knowledge of the atmospheric aerosol. The general conclusion is that the size distribution of atmospheric particles is not self-preserving as a result of a balance between generating and eliminating mechanisms-because the residence times of particles are too short-but that it is a reflexion of the action of various sources of aerosols.

An alternative to counting aerosol particles is to form a jet surrounded with clean air which is directed towards a microscope objective so that lateral displacements can be accurately measured. Thermophoresis and diffusiophoresis of the particles can be measured. Variation of the supersaturation of the clean air sheath makes it possible to study the formation of visible aerosol upon condensation nuclei in the aerosol jet.

The session on production and measurement of aerosols included descriptions of three new thermal generators. One used helium as a carrier gas, with nuclei of sodium chloride, and evaporated the aerosol material from a film of liquid running down the inside wall of a vertical tube; another made an aerosol of solid particles of carnauba wax and the third employed high frequency induction heating to generate silver aerosols having diameters between 0.002 and $0.1 \mu \mathrm{m}$. Size analysis by light scattering and diffusion, the growth of particles by condensation and a dust generator were also discussed.

Papers on size analysis and shape factors covered automation and the use of fibre optics for scanning projected areas. There was a study of mechanical size separation arid an aerosol spectrometer working by sedimentation into a stream of clean air adjacent to the sampled aerosol was described in detail; it was applied to industrial dusts, including asbestos fibres, and to testing sizeselecting samplers and filters.

Two papers on shape factors were based on experiments with a spiral centrifuge aerosol spectrometer; one dealt with latex sphere aggregates and fibres and the other with dusts of coal, $\mathrm{UO}_{2}$ and $\mathrm{ThO}_{2}$.

Inhaled aerosols were the subject of calculations to try and show the differences in deposition between spheres and ellipsoids and the retention of insoluble particles in animals of different species in terms of lung clearance and storage in the tracheobronchial lymph nodes. Calculations were also presented which were aimed at finding the effects of zero gravity and of hygroscopic particles. Experiments in human breathing of $0.5 \mu \mathrm{m}$ diameter particles showed that deposition decreases when the reserve volume is raised. The inhalation of dust from polyurethane foam was found to produce carcinoma in rats. Experiments with various kinds of asbestos fibres indicate that the sedimentation rate depends mainly on fibre diameter, and when this exceeds $3 \mu \mathrm{m}$ penetration to the alveolated regions of the human lungs is improbable unless the fibres are open, fluffy bundles of fibrils which have a low sedimentation rate.

The last session of the conference covered sampling methods for use in occupational hygiene. Selective sampling is achieved by cyclones in a two stage device which can be matched to deposition in the mouth or nose and in the tracheobronchial regions; another device uses a cyclone followed by a bubbler which was believed to match alveolar deposition. The preparation of filters from aluminium oxide which have a pore size of $0.025 \mu \mathrm{m}$ is described; they withstand temperatures up to $700^{\circ} \mathrm{C}$ so that dust deposits can be incinerated. By using these filters in mines it was shown that fine dust below $1 \mu \mathrm{m}$ diameter does not contribute appreciably to the mass of the respirable fraction $(50 \%$ cut at $5 \mu \mathrm{m})$ but that it accounts for some $20 \%$ of the surface area.

The remaining two chapters deal with the sampling of urban aerosols and of viable particles. The former is a plea for a less general approach to the formulation of quality criteria and the latter describes the microthread technique for obtaining a "captive aerosol" and the disclosure of an atmospheric factor toxic to microorganisms which it has led to.

C. N. Davies

\section{Statistics in Russian}

Russian - English / English - Russian Glossary of Statistical Terms. By Samuel Kotz. Based on A Dictionary of Statistical Terms by M. G. Kendall and W. R. Buckland. Pp. vii +87 . (Edinburgh: Oliver and Boyd, 1972.) £3.

THIS dictionary was originally envisaged as a sixth volume to the combined foreign-language glossary in the now standard Dictionary of Statistical Terms of M. G. Kendall and W. R. Buckland; however, in the course of compilation it developed into a new and valuable work in its own right.

Unlike the best-known existing statistical glossaries for Russian, this volume contains both Russian-English and English-Russian sections, the latter containing some 2,500 terms, the former being somewhat larger. Thus, although it will prove more valuable to the western reader wishing to read Russian statistical material in the original, the English-Russian section is sufficiently comprehensive for the purposes of the English-speaking statistician who may need to write an occasional letter in Russian to a colleague or library in the Soviet Union, asking, say, for additional material in a given field. It would probably not, however, be sufficient for the compilation or translation of scientific papers into Russian, although it would, again, prove useful for the writing of the Russian-language abstract now so often required for international conferences.

Russian statistical vocabulary differs somewhat from English, and some terms have no exact equivalent. Thus, on a single page (p. 82) we find five terms (Sturges's rule, sub-normal-dispersion, substitute F-ratio, superfluous 